\title{
Querying Similarity in Metric Social Networks
}

\author{
Jan Sedmidubský, Stanislav Bartoň, Vlastislav Dohnal, and Pavel Zezula \\ Masaryk University \\ Brno, Czech Republic \\ [xsedmid, xbarton, dohnal, zezula]@fi.muni.cz
}

\begin{abstract}
In this paper we tackle the issues of exploiting the concepts of social networking in processing similarity queries in the environment of a P2P network. The processed similarity queries are laying the base on which the relationships among peers are created. Consequently, the communities encompassing similar data emerge in the network. The architecture of the presented metric social network is formally defined using the acquaintance and friendship relations. Two version of the navigation algorithm are presented and thoroughly experimentally evaluated. Finally, learning ability of the metric social network is presented and discussed.
\end{abstract}

\section{Introduction}

The area of similarity searching is a very hot topic for both research and commercial applications. Current data processing applications use data with considerably less structure and much less precise queries than traditional database systems. Examples are multimedia data like images or videos that offer queryby-example search, product catalogs that provide users with preference-based search, scientific data records from observations or experimental analyses such as biochemical and medical data, or XML documents that come from heterogeneous data sources on the Web or in intranets and thus does not exhibit a global schema. Such data can neither be ordered in a canonical manner nor meaningfully searched by precise database queries that would return exact matches.

This novel situation is what has given rise to similarity searching, also referred to as content-based or similarity retrieval. The most general approach to similarity search, still allowing construction of index structures, is modeled in metric space. Many index structures were developed and surveyed recently [12]. However, the current experience with centralized methods [6] reveals a strong correlation between the dataset size and search costs. Thus, the ability of centralized indexes to maintain a reasonable query response time when the dataset multiplies in size, its scalability, is limited. The latest efforts in the area of similarity searching focus on the design of distributed access structures which exploit more computational and storage resources $[2,7,4,3]$. Current trends are optimizing and tuning the well known distributed structures towards better utilization of the available resources.

Another approach to design the access structure suitable for large scale similarity query processing that is introduced in this paper emerges from the notion of 
social network. A social network is a term that is used in sociology since the 1950s and refers to a social structure of people, related either directly or indirectly to each other through a common relation or interest [10]. Using this notion, our approach places the peers of the distributed access structure in the role of people in the social network and creates relationships among them according to the similarity of the particular peer's data. The query processing then represents searching for a community of people, i.e., searching for peers related by a common interest, for example, maintaining similar data.

Using this data point of view our designed metric social network is a cognitive knowledge network according to the terminology stated in [9] that is described as who thinks who knows what where it is not who you know but it is what who you know knows. This means that the network links are created on the basis of the particular peers' knowledge (stored data) rather than on being acquainted with other peers. As for the navigation, social networks exhibit the small world network topology [11] where most pairs of nodes are reachable by a short chain of intermediates - usually the average pairwise path length is bound by a polynomial in $\log n$. Therefore it is anticipated that a small amount (around six) of transitions will be needed to find the community of peers holding the answer to a query posed at any of the participating peers in the network.

Unlike the usual access structures that retrieve a total answer to each query, the presented approach focuses on retrieving the substantial part of the answer yet with partial costs compared to the usual query processing. The concepts of social networking towards the approximative query processing in large scale data have already been introduced in related works $[1,8]$.

The paper is structured in following sections. In Section 2, the architecture of the access structure is detailed. In Section 3, the preliminary experimental results are reviewed to present the nature and behavior of the proposed approach. Finally, the conclusions are drawn in Section 4.

\section{Architecture}

Our social network comprises of entities as usual - vertices and edges representing relationships among them. The relationships between two entities are of two types: the friendship and a relation of acquaintance. The relationships identified among the entities in the graph always relate to a particular query processed by the network and its retrieved answer.

\subsection{Vertices of the Social Network}

The vertex itself, besides the assigned piece of data, remembers also the history of the queries that it has been asked. To each query the recognized set of friends and acquaintances is also remembered for future optimization of a similar query processing. So, a network peer $P$ is $P=(D, H)$ where $D=\left\{o_{1}, \ldots o_{l}\right\}$ represents the assigned piece of data and $H=\left\{h_{1}, \ldots h_{m}\right\}, h_{i}=\left(Q, L_{P}^{A c q}(Q), L_{P}^{F r i}(Q)\right)$ represents the history of queries with the pair of ordered lists of retrieved acquaintances and friends regarding the particular query $Q$. For example as a query a 
usual range query can be considered: $R(q, r)$ where $q$ is a query object and $r$ is a predefined range. The peer which is asked to answer a query $Q$ in the social network is denoted as $P_{\text {start }}$. Consequently, partial answers $A_{P_{i}}(Q)$ are passed from the peers $P_{i}$ of the network to $P_{\text {start }}$. The final answer is $A(Q)=\bigcup_{i=1}^{n} A_{P_{i}}(Q)$ where $n$ denotes the total number of peers that participated on the answering.

\subsection{Measuring Quality of the Query Answer}

As we have seen, the total answer can be divided into pieces regarding the peers that participated on the total answer to a query $Q$. To distinguish which peer answered better, the quality is measured by defining a quality measuring function $\operatorname{Qual}\left(A_{P_{i}}(Q)\right)$. It returns a quality object $q_{i}$ that represents the quality of the peer's answer. Since this object is not necessarily a number, we also define a function to compare two quality objects:

$$
\text { compare }_{\text {qual }}\left(q_{1}, q_{2}\right)= \begin{cases}-1 & q_{1} \text { is worse than } q_{2} \\ 0 & q_{1} \text { is same as } q_{2} \\ 1 & q_{1} \text { is better than } q_{2}\end{cases}
$$

The quality of the total answer is determined by applying the quality measuring function on $A(Q)$. An ordering $\preceq$ which we call the q-ordering is defined on the peers' answers $A_{P_{1}}(Q), \ldots, A_{P_{n}}(Q)$ in $A(Q)$ according to their qualities. The quality objects are then $q_{i}=\operatorname{Qual}\left(A_{P_{i}}(Q)\right)$. The sequence of peers' answers is ordered according to the q-ordering when the following holds:

$$
i_{1} \ldots i_{n}: q_{i_{a}} \preceq q_{i_{b}} \Leftrightarrow a<b \Leftrightarrow \text { compare }_{\text {qual }}\left(q_{i_{a}}, q_{i_{b}}\right) \neq 1
$$

Intuitively, when the peers are ordered with respect to the position of their answers in the q-ordered set of partial answers, they are ordered by their ability to answer the particular query $Q$.

\subsection{Acquaintance and Friendship Relations}

As we mentioned earlier, we distinguish two relationships in our social network. Firstly, the relationship of friendship represents the similarity of nodes - two nodes give a similar answer to same query. Secondly, the relationship of acquaintance denotes that the target of the relationship took part in the answer passed to the recipient. In the following, we will use the answer size in objects as the measure of quality, i.e., $\operatorname{Qual}\left(A_{P}(Q)\right)=\left|A_{P}(Q)\right|$.

A set of acquaintances for a given query $Q$ is defined as a set of participating peers in the total answer $A c q(Q)=\left\{P \mid A_{P}(Q) \neq \emptyset\right\}$. Friends are identified in the set of acquaintances as peers that at which the similarity of the data kept could be anticipated: $\operatorname{Fri}(Q)=\left\{P|| A_{P}(Q)|>c \cdot| A(Q) \mid\right\}$. The friendship relationship according to the particular query $Q$ is assigned only to those peers that contributed to the answer with a significant partial answer, which is expressed by the positive-value constant $c$. Then, according to a query $Q$, a peer $P$ is an acquaintance $P^{A c q}(Q) \Leftrightarrow P \in A c q(Q)$. Similarly, with respect to a query $Q$, a peer $P$ is a friend $P^{F r i}(Q) \Leftrightarrow P \in \operatorname{Fri}(Q)$. Intuitively, the set of acquaintances 
and friends of each peer that took part in the query processing can be determined using following functions:

$$
\begin{aligned}
& \operatorname{Acq}_{P}(Q)= \begin{cases}\operatorname{Acq}(Q) & P \in \operatorname{Fri}(Q) \vee P=P_{\text {start }}(Q) \\
\emptyset & \text { otherwise }\end{cases} \\
& \operatorname{Fri}_{P}(Q)= \begin{cases}\operatorname{Fri}(Q) & P \in \operatorname{Fri}(Q) \\
\emptyset & \text { otherwise }\end{cases}
\end{aligned}
$$

The edges of the social network are then defined by the two relations defined among the peers - vertices of the network for a given query $Q$ :

$$
\begin{gathered}
P_{1} \sim_{Q}^{A c q} P_{2} \Leftrightarrow P_{2} \in \operatorname{Acq}_{P_{1}}(Q) \\
P_{1} \sim{ }_{Q}^{F r i} P_{2} \Leftrightarrow P_{1} \in \operatorname{Fri}_{P_{2}}(Q), \quad P_{1} \in \operatorname{Fri}_{P_{2}}(Q) \Leftrightarrow P_{2} \in \operatorname{Fri}_{P_{1}}(Q)
\end{gathered}
$$

The edges in the history are stored as two lists of peers ordered according to the q-ordering of the partial answers in $A(Q)$ with respect to their qualities. The ordering respects the position of the peer's answer in the q-ordered $A(Q)$. Each of the lists comprises of pairs of a peer $P_{i}$ and a quality object $q_{i}=\operatorname{Qual}\left(A_{P_{i}}(Q)\right)$ :

- $L_{P}^{F r i}(Q) \ldots$ a list of friends of the peer $P$ for a query $Q$ sorted by $q$-ordering. - $L_{P}^{A c q}(Q)$... a list of acquaintances of $P$ for $Q$ sorted by $q$-ordering .

\subsection{Navigation}

The query processing using the social network follows the common world concepts for searching. Basically, the best acquaintance regarding the particular subject is located and then his friends are contacted to return their part of the answer to the querist.

The acquaintances are contacted firstly because they represent the entities that have answered before. Initially, the $P_{\text {start }}$ goes through its history of processed queries and finds the most similar query $Q^{\prime}$ to the query $Q$ that is processing now. For query $Q^{\prime}$, also the lists of acquaintances and friends are retrieved from the history. The query $Q$ is then forwarded to the best acquaintance. This concept is formalized in Algorithm 1. In general, the query can be passed to more then one acquaintance, it depends on the particular navigation algorithm implementation. The process of the query forwarding can be repeated more times to find the peer that is most promising to hold the searched data. At each peer, a different query can be retrieved from the history as the most similar to $Q$. The stop condition of the query forwarding is when the contacted peer's quality is better than any of his acquaintances to which it could pass the query.

When the best acquaintance regarding the particular query is found, it returns its part of the query answer to the querist. Then it looks up in the history for the most similar query and retrieves the set of friends associated with that query and forwards the query $Q$ to them as described in Algorithm 2. The query is passed also to friends because it is supposed that they hold similar data which will form substantial parts of the query answer $A(Q)$. After contacting, the peers return their partial answers $A_{P_{i}}(Q)$ to $P_{\text {start }}$. 

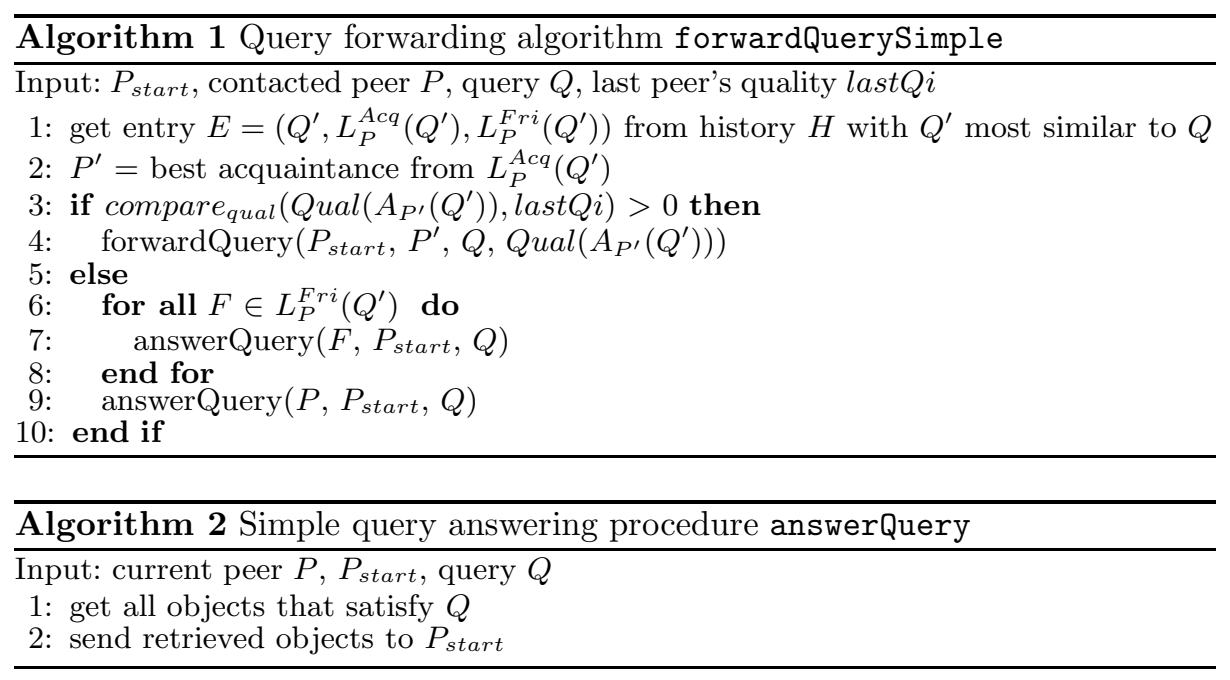

\section{Experimental Results}

In this section, we present an experimental evaluation of the proposed distributed access structure for searching in metric data. The experiments have been conducted on two datasets represented by vectors having three and fortyfive dimensions respectively. The 45-D vectors represent extracted color image features compared using a quadratic-form distance. The distribution of the dataset is quite uniform and such a high-dimensional data space is extremely sparse. The 3 -D vectors were obtained as the three most-important dimensions of $45-\mathrm{D}$ vectors. The number of vectors in each dataset was 100,000 .

\subsection{Network Initialization}

The peers in the network have been created using the distributed version of the M-tree [5] indexing structure - each peer corresponds to one M-tree leaf node. Besides the data distribution among peers, the M-tree was also used to evaluate the precise total answer to queries.

Firstly, the distributed M-tree is created on the provided dataset. Next, the peers are assigned their pieces of data. ${ }^{1}$ The edges in the network are then acquired by posing queries to the created M-tree and processing its answer retrieved from the peers in the network. This process is called learning.

Intuitively, the longer the learning process is the better the social network query processing is afterwards. In the set of experiments regarding the recall and costs of the query processing using the social network, the learning process comprised of 500 queries processed using the M-tree.

${ }^{1}$ For both datasets, the M-tree consisted of 47 leaf nodes - the data have been distributed among 47 peers. 

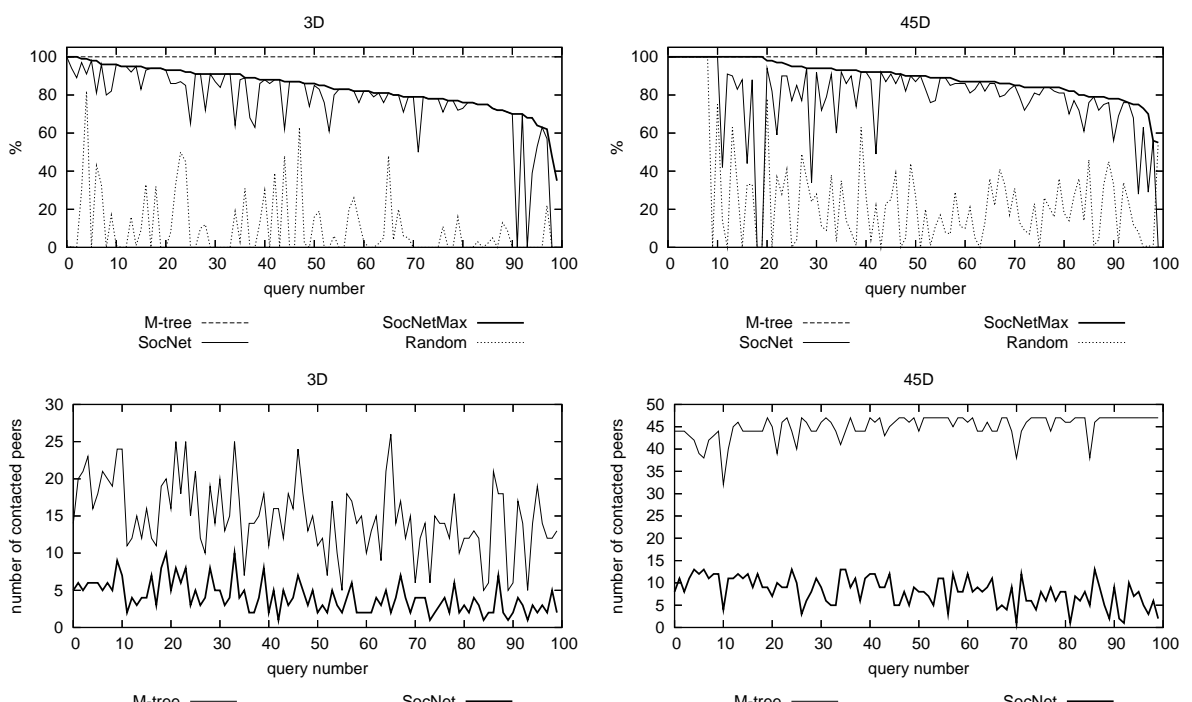

Fig. 1. Results of one hundred queries processed using the social network compared to the total answer of the M-tree: (top row) Recall and (bottom row) Costs.

\subsection{Recall and Costs}

To demonstrate the properties of querying process in the social network, we used the basic variant of algorithm described in Section 2.4. A recall of one hundred queries processed using the built social network is presented in Fig. 1. The queries represent range queries with randomly picked objects with a fixed radius of 200 and 2,000 for the 3-D and 45-D data, respectively. These radii have been chosen because they returned on average $3-5 \%$ of data.

The queries in Fig. 1 are ordered in a descending manner with respect to the SocNetMax recall values. Both figures demonstrate the percentage of the total answer retrieved using the social network. The recall of SocNetMax value denotes a maximal part of the total answer that could have been retrieved contacting the same amount of peers that the social network (SocNet) did, i.e. if the social network has contacted eight peers, the SocNetMax represents the percentage of objects from the total answer represented by the best eight peers. Finally, Random represents the percentage of the total answer retrieved from the randomly picked peers which amount is the same as the social network has contacted.

The costs of a query processed presented in Fig. 1 are defined as the amount of peers that are contacted in order to answer the query - contacted by the answerQuery procedure. We can see that the amount of the peers contacted per query by SocNet is substantially smaller than the numbers of the M-tree in the case of the 3-D data. The gap between those two curves is even greater for the 45 -D data. This is caused by the worse clusterability of the latter dataset, since 

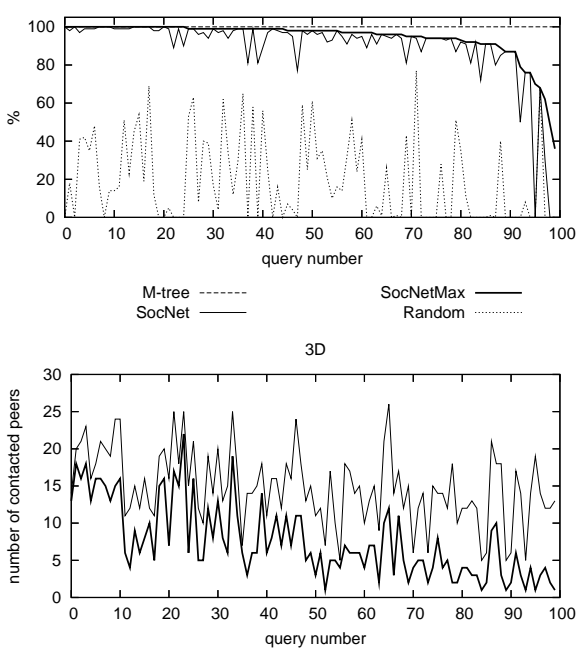

M-tree - SocNet -

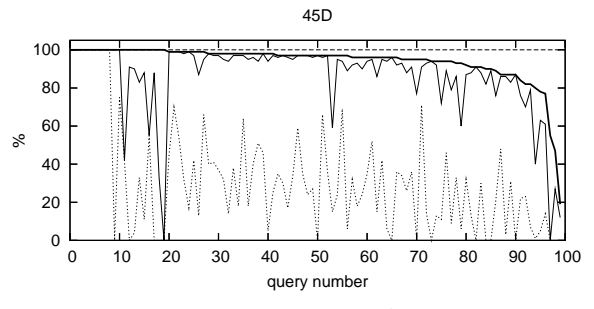

M-tree -------

SocNetMax

45D

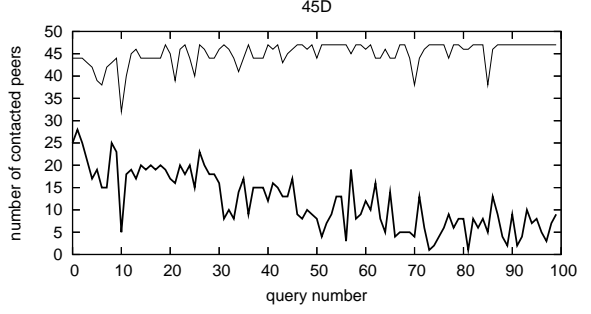

M-tree - SocNet -

Fig. 2. Results of queries processed by the social network using the forwardQueryFOAF navigation algorithm: (top row) Recall and (bottom row) Costs.

distance between any pair of the objects is more or less the same in this high dimensional data.

The fluctuations in both figures are strictly related, i.e. recall is decreasing with the decreasing number of peers used to answer queries. Such a behavior of SocNet is caused by insufficient social information available to some peers. For the 45 -D data, the recall exhibits higher fluctuations than for the 3-D data, which can be attributed to the fact that the complete result is spread over more nodes.This is also proportional to the ratio between the number of contacted peers by the M-tree and by SocNet. An improved query routing strategy tries to eliminate such instability and is presented in the following section.

\subsection{Friend-of-a-Friend Query Forwarding}

The great differences between SocNet and SocNetMax in Fig. 1 present a fair amount of instability of the SocNet access structure. We have enhanced the navigation algorithm towards the greater stability of the gained results yet with the emphasis on sustaining the low query processing costs. The enhanced query answering procedure is described in Algorithm 3. Despite calling the enhanced answering routine, the query forwarding procedure (Algorithm 1) remains the same. We will refer to this enhanced algorithm as the forwardQueryFOAF.

Using the forwardQueryFOAF navigation algorithm, the set of peers that participate in the final answer grows because the contacted community of the similar peers grows larger. The trends of the results gained in terms of recall and costs 


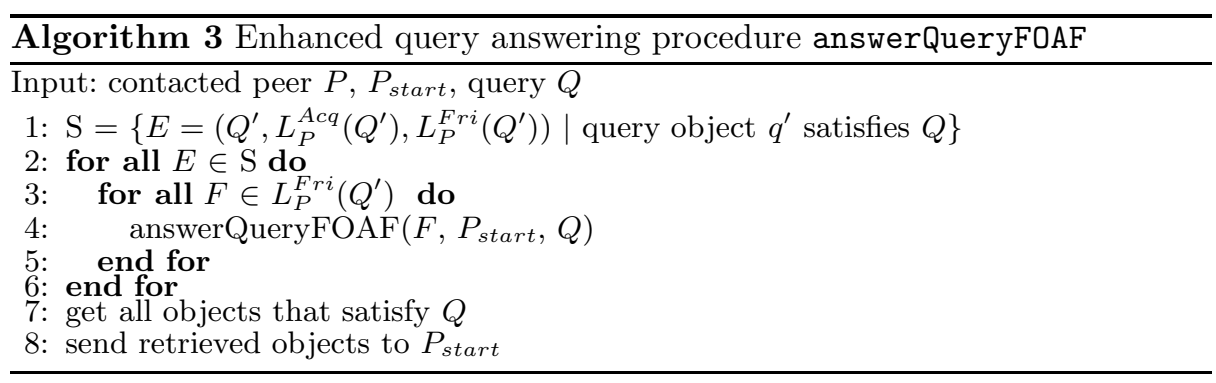

can be read from Fig. 2. The queries on which we have measured the properties of the enhanced navigation algorithm are the same as those on which we measured the simple algorithm in the previous subsection.

\subsection{Learning Abilities}

In the introduction to the architecture of the designed social network, we have mentioned that the qualities of answers to each query are being stored in peers that participated in the query processing, i.e., the social network is being updated. For the structure's evaluation purposes, this feature was disabled during the experiments conducted in the previous section.

In this section, we demonstrate the ability of the designed social network to learn itself towards better query processing. The experimental results are presented using only the 3-D dataset because the results gained on the other dataset were very similar.

To measure the ability to learn, a fixed testing set consisting of twenty randomly picked queries was used. The method is that after processing each $50^{\text {th }}$ query, the self-adaptability of the social network is turned off and the recall and costs on the testing set is examined. Progress of average recall and cost values on the testing queries during time represents the ability of the social network to learn. Two initial states of the network were used. Firstly, the social network is well trained using 250 queries processed by the M-tree. Secondly, this state represents a state of the network that is not trained yet. The edges in the network were created randomly and assigned to processed queries by the M-tree.

Figure 3 demonstrates the evaluation of the training abilities of the social network using the forwardQuerySimple algorithm and forwardQueryFOAF algorithm for navigation. In this figure, the curves recall 1 and costs 1 represent results initiated in the well-trained social network. On contrary, the recall 2 and costs 2 demonstrate the same abilities on the randomly initialized network. Also the costs of the M-tree are presented. The cost values are presented as a proportion of connected peers to the total amount of peers in the network. The presented results demonstrate the inability of the social network equipped with the forwardQuerySimple to refine its social information towards more precise query answering. This is caused by the insufficient overall quality of the 

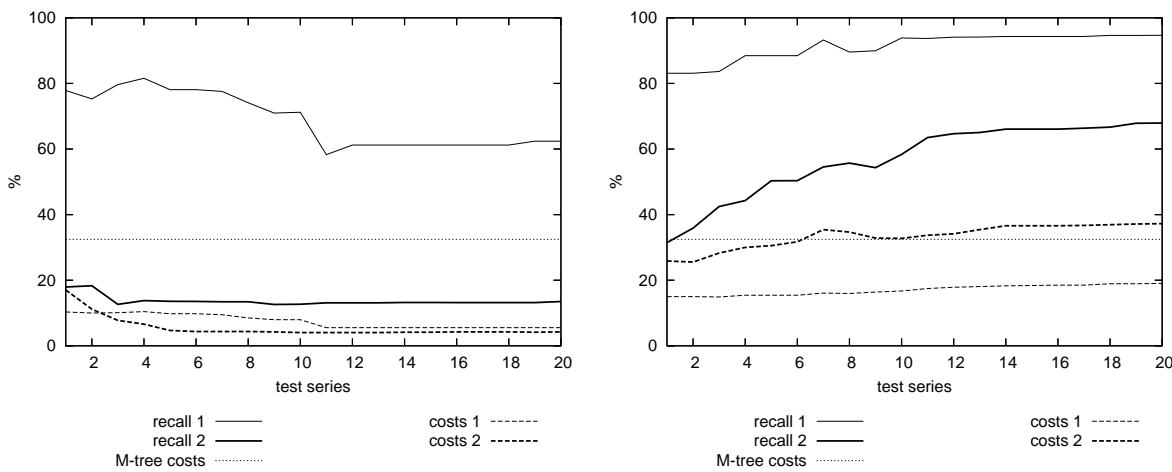

Fig. 3. Learning abilities of the social network access structure: (left) using forwardQuerySimple and (right) using forwardQueryFOAF.

answer retrieved from the network. This inability is underscored by the poor results when deployed on the random initial state social network resulting.

On the other hand, using the forwardQueryFOAF algorithm to process the queries in the social network yields better results proved by the rising curve representing improving recall values starting at both well-trained and random initial states of the social network. This fact proves that the algorithm and the training abilities are communicating vessels since the more precise answers the better the learning ability is.

\section{Concluding Remarks and Future Work}

Distributed processing of similarity queries currently attracts a lot of attention because of its inherent capability of solving the issue of data scalability. We have proposed an approach based on social networking which is able to answer any similarity query modelled using metric space paradigm. The principle exploited in this proposal models social relationships with regards to specific queries. As a result, a multigraph is created in which individual communities sharing similar data can be identified. The presented experiment trails confirm suitability and auspiciousness of such approach. Moreover, the network with the enhanced navigation algorithm is able to evolve autonomously while improving quality of query results.

For future work, various aspects of navigation strategies will be deeply studied in order to design more sophisticated and possibly self-adapting policies. The peers in our social networks were assigned with data objects based on the Mtree clustering principle. Influence of such data partitioning will be verified. Also dynamicity in the sense of peers' joining and leaving the network will be investigated. This is also related to the dynamicity from the data point of view where the data content of individual peer can change, which invalidates relationships established in the network so far. 


\section{Acknowledgements}

Partially supported by: the EU IST FP6 project 045128 (SAPIR), the national research project 1ET100300419, the Czech Grant Agency project 201/07/P240.

\section{References}

1. R. Akavipat, L.-S. Wu, F. Menczer, and A.G. Maguitman. Emerging semantic communities in peer web search. In P2PIR '06: Proceedings of the international workshop on Information retrieval in peer-to-peer networks, pages 1-8, New York, NY, USA, 2006. ACM Press.

2. Farnoush Banaei-Kashani and Cyrus Shahabi. SWAM: A family of access methods for similarity-search in peer-to-peer data networks. In CIKM '04: Proceedings of the Thirteenth ACM conference on Information and knowledge management, pages 304-313. ACM Press, 2004.

3. Michal Batko, David Novak, Fabrizio Falchi, and Pavel Zezula. On scalability of the similarity search in the world of peers. In Proceedings of First International Conference on Scalable Information Systems (INFOSCALE 2006), Hong Kong, May 30 - June 1, pages 1-12. ACM Press, 2006.

4. Matthias Bender, Sebastian Michel, Peter Triantafillou, Gerhard Weikum, and Christian Zimmer. MINERVA: Collaborative P2P search. In VLDB '05: Proceedings of the 31st international conference on Very large data bases, pages 1263-1266. VLDB Endowment, 2005.

5. Paolo Ciaccia, Marco Patella, and Pavel Zezula. M-tree: An efficient access method for similarity search in metric spaces. In Matthias Jarke, Michael J. Carey, Klaus R. Dittrich, Frederick H. Lochovsky, Pericles Loucopoulos, and Manfred A. Jeusfeld, editors, Proceedings of the 23rd International Conference on Very Large Data Bases (VLDB 1997), Athens, Greece, August 25-29, 1997, pages 426-435. Morgan Kaufmann, 1997.

6. Vlastislav Dohnal, Claudio Gennaro, Pasquale Savino, and Pavel Zezula. D-index: Distance searching index for metric data sets. Multimedia Tools and Applications, 21(1):9-33, 2003.

7. Prasanna Ganesan, Beverly Yang, and Hector Garcia-Molina. One torus to rule them all: Multi-dimensional queries in $\mathrm{P} 2 \mathrm{P}$ systems. In WebDB '04: Proceedings of the 7th International Workshop on the Web and Databases, pages 19-24, New York, NY, USA, 2004. ACM Press.

8. Alessandro Linari and Gerhard Weikum. Efficient peer-to-peer semantic overlay networks based on statistical language models. In P2PIR '06: Proceedings of the international workshop on Information retrieval in peer-to-peer networks, pages 9-16, New York, NY, USA, 2006. ACM Press.

9. Peter R. Monge and Noshir S. Contractor. Theories of Communication Networks. Oxford University Press, April 2003.

10. Stanley Wasserman, Katherine Faust, and Dawn Iacobucci. Social Network Analysis : Methods and Applications (Structural Analysis in the Social Sciences). Cambridge University Press, November 1994.

11. D. J. Watts and S. H. Strogatz. Collective dynamics of 'small-world' networks. Nature, 393(6684):440-442, June 1998.

12. Pavel Zezula, Giuseppe Amato, Vlastislav Dohnal, and Michal Batko. Similarity Search: The Metric Space Approach, volume 32 of Advances in Database Systems. Springer, 2005. 